ISSN: $1130-3743$

\title{
LÍNEAS DE DESARROLLO Y FUNDAMENTACIÓN EN EL CAMPO DE LA DEONTOLOGÍA DE LAS PROFESIONES EDUCATIVAS
}

\author{
SOME LINES OF DEVELOPEMENT AND FOUNDATION \\ IN THE FIELD OF EDUCATIONAL PROFESSIONE ETHICS
}

GONZALO JOVER OLMEDA

Universidad Complutense. Departamento de Teoría e Historia de la Educación. Facultad de Educación. Edificio La Almudena, $P^{\circ}$ de Juan XXIII s/n., 28040 Madrid.

RESUMEN

A la vista del interés que están suscitando las cuestiones y regulaciones deontológicas en nuestro país, y que ha llevado a algunas instituciones y organizaciones profesionales a proponer códigos deontológicos para los profesores, este artículo pretende hacer de la deontología en el campo de las profesiones educativas un objeto de investigación pedagógica. En primer lugar, se expone la tradición normativa en las áreas de la enseñanza, administración educativa, intervención psicopedagógica, educación social e investigación. Más tarde se establecen las líneas principales para una fundamentación de la deontología profesional desde un triple plano: sociológico, ético-jurídico y pedagógico. Finalmente, se sugiere la posibilidad de entender los Códigos deontológicos como elementos de proyectos educativos.

\section{SUMMARY}

Nowadays professional ethics is generating great attention and expectancy. Institutions and professional organizations in Spain have recently proposed. Codes 
of Ethics in teaching. According to that new interest, this paper tries to make professional ethics in the field of education a subject of educational research. First, the paper focuses in the normative tradition in the areas of teaching, educational administration, counselling and special education, social education, and research. Secondly, a framework is proposed allowing a foundation of professional ethics from the point of view of sociology, ethics and law, and pedagogy. Finally the author suggests to understand codes of ethics as elements of educational projects.

\section{INTRODUCCIÓN}

En los últimos años la cuestión de la deontología viene generando dentro y fuera de nuestro país un gran interés. Éste ha sobrepasado el marco de las profesiones clásicas y ha llevado a nuestros colectivos profesionales a establecer códigos deontológicos. Al mismo tiempo, se ha convertido en algo recurrente insistir en la necesidad de acercar más la ética a la política, y se proponen códigos de conducta para la Administración y los partidos políticos. Algunos medios de comunicación se dotan, asimismo, de normas deontológicas. Y en economía cunde el lema good ethics is good businnes,... Lipovetsky ha lamentado la visión instrumentalista de la ética que esconde a menudo este recurso a la deontología:

"La moda de los códigos o cartas éticas no tiene nada de idealista, está sostenida en lo más profundo por la creencia de que la ética es esencial para el éxito comercial y financiero, ethics pays: los dos tercios de la élite profesional consideran ya que la moral contribuye al éxito de la empresa. Si la caridad-espectáculo explota la moral del sentimiento, la business ethics se basa en la moral del interés bien concebido: lo que caracteriza a nuestra época no es la consagración de la ética, sino su instrumentalización utilitarista en el mundo de los negocios" (Lipovetsky, 1994, pp. 249-250).

Más que en la revalorización del componente ético de la vida social, la apelación a las regulaciones deontológicas puede llegar a convertirse, de este modo, en un signo de desorientación y desconfianza:

"Vivimos en un mundo plural, sin ideologías sólidas y potentes, en sociedades abiertas y secularizadas, instaladas en el liberalismo económico y político. El consumo es nuestra forma de vida. Desconfiamos de los grandes ideales porque estamos asistiendo a la extinción y fracaso de la utopía más reciente. Nos sentimos como de vuelta de muchas cosas, pero estamos confusos y desorientados, y nos sacude la urgencia y la obligación de emprender algún proyecto común que dé sentido al presente y oriente el futuro. Hemos conquistado el refugio de la privacidad y unos derechos individuales, pero echamos de menos una vida pública más aceptable y más digna de crédito" (Camps. 1990, pp. 7-8).

Diariamente, por otra parte, la prensa nos informa de problemas éticos de convivencia en las instituciones educativas. Estas son, a título de muestra, algunas 
de las noticias con las que pudimos desayunarnos durante 1994: “Crece el racismo en la escuela: casi un tercio de los alumnos de Medias es partidario de expulsar a los gitanos; “Un catedrático fotocopió autobiografías de sus alumnos tras garantizarles la confidencialidad"; "Los universitarios no se atreven a denunciar a los profesores que se apropian de sus trabajos"; "El director de un colegio sancionado por racista"; "Un profesor de un colegio público es acusado de cometer abusos sexuales"; "La crueldad en el cole; el maltrato sistemático entre escolares, una realidad ignorada que empieza a preocupar en España"; "Un profesor expedientado por posible prevaricación administrativa, al ser denunciado por sus compañeros de aprobar a su hija con sobresaliente en dos cursos de doctorado y colocarla como profesora asociada"; "Los docentes reclaman más protección jurídica de la administración, ante el incremento de demandas de responsabilidad civil....

El clima social que configuran este tipo de factores explica que también en el campo de la enseñanza y de la educación se haya despertado la misma sensibilidad hacia la necesidad de una regulación ética. La propia Comisión de la Unión Europea resalta en una de sus comunicaciones cómo «la calidad y ética del cuerpo docente son motivo de preocupación general" (Comisión de las Comunidades Europeas, 1993, p. 4). A finales de 1992, el Consejo Escolar Catalán, aprobaba el proyecto de un código deontológico para profesores (Consejo Escolar Catalán, 1993), y algo más tarde el Consejo General de Colegios Oficiales de Doctores y Licenciados en Filosofía y Letras y en Ciencias sometía a consideración el borrador de un Código deontológico docente (Consejo General de Colegios Oficiales de Doctores y Licenciados en Filosofía y Letras y en Ciencias, 1994).

A la vista de este nuevo interés, se trata, pues, ahora de hacer de la deontología profesional también un objeto de investigación pedagógica. Ya en un trabajo anterior se sugirieron a este respecto algunas posibles vías de investigación, en lo que se refiere tanto al desarrollo en tradición normativa y contenido regulativo de la deontología profesional, como a la fundamentación, posibilidades y límites del planteamiento deontológico (Jover, 1991). Dos grandes núcleos de problemas entre los que no siempre existe la suficiente relación, tal como señala Kultgen al inicio de su obra Etica y profesionalismo, cuando subraya la falta de comunicación entre la literatura sobre regulaciones deontológicas en campos específicos y los estudios sociológicos sobre profesionalización, entre la dimensión normativa y la dimensión descriptiva, y entiende que el análisis de la ética profesional exige de una filosofía social que vincule ambas dimensiones (Kultgen, 1988, pp. 6-7). En un simposio sobre ética profesional organizado en nuestro país, Hortal Alonso venía a resaltar la misma brecha, indicando que "O encontramos la forma de empalmar esas dos perspectivas, el texto y el contexto, o lo único que haremos será generar una ética para nuestras charlas de café y para desahogarnos de nuestras frustraciones. La realidad irá por otro sitio, ajena a nuestros comentarios y desahogos" (Hortal, 1994, p. 56). En lo que sigue voy a tratar, por tanto, de abarcar ambas

1. Diario 16, 12-1-94; El País, 30-1-94; El País, 6-2-94; Diario 16, 16-3-94; El País, 29-3-94; El País, 19-6-94; El País, 20-10-94; ABC, 25-10-94. 
GONZALO JOVER OLMEDA

LÍNEAS DE DESARROLLO Y FUNDAMENTACIÓN EN EL CAMPO DE LA DEONTOLOGÍA

coordenadas. En un primer momento haré una revisión de la tradición deontológica en algunas de las principales profesiones educativas, para más tarde trazar un marco de fundamentación en un sentido más amplio que aúne, a su vez, lo descriptivo y lo normativo.

\section{LA TRADICIÓN DEONTOLÓGICA EN EL CAMPO DE LAS PROFESIONES EDUCATIVAS.}

Pueden identificarse cinco grandes áreas de atención deontológica en lo que cabe denominar el campo amplio de las profesiones de educación: enseñanza, administración educativa, intervención psicopedagógica, educación social e investigación. Me centraré sobre todo en la tradición normativa en deontología profesional que ha venido desarrollándose en estas áreas en Estados Unidos, a la que tuve ocasión de acercarme durante un período de estancia como Visiting Scholar en la Universidad de Boston.

Acerca de la primera de ellas, el trabajo de recopilación publicado por Herring en 1988 da idea, más allá del manido tópico del "publica o perece", de la magnitud del interés que internacionalmente suscitan las distintas cuestiones en torno a la ética de la actividad docente (Herring, 1988) ${ }^{2}$. En materia de regulación deontológica, las asociaciones americanas de profesores cuentan con una tradición cuyos primeros precedentes se remontan a hace un siglo. El primer código de alcance estatal fue adoptado en 1896 por la Georgia Education Association, iniciativa en la que se sumaría otras asociaciones estatales en los años veinte. En el ámbito federal, la National Education Association estableció en 1924 un comité de ética profesional, que formuló un código adoptado por la Asociación en 1929, experimentando posteriormente diversas reelaboraciones, hasta la última de 1975. Por su lado, la American Association of university Professors, fundada en 1915, establecía en 1966 una Declaración de Etica Profesional, suscitándose en las últimas décadas una apreciable producción bibliográfica en torno a la Academic Etbics y, en general, a la ética aplicada a la enseñanza superior ${ }^{3}$. Durante los años ochenta y comienzo de los noventa, se produce un nuevo desarrollo y diversificación del interés ético y deontológico por especialidades, niveles educativos, etc. Organizaciones docentes y asociaciones profesionales en el campo de los estudios sociales, educación infantil, educación para la salud o tecnología educativa, adoptan, revisan o elaboran códigos éticos.

Respecto a la segunda de las áreas indicadas, en un artículo en The American School Board Journal resaltaba Natale algunos titulares de prensa sobre corrupción en el campo de la administración escolar, y señalaba cómo los mismos represen-

2. Formulaciones más recientes de interés, las representan obras como las de Goodlad, Soder y Sirotnik (1990); Sockett (1993); Strike y Ternasky (1993); o los números monográficos del Journal of Thought (1987); Jorunal of Teacher Education (1986 y 1991) o Journal of Moral Education (1993).

3. Véase, por ejemplo: The Journal of Higher Education (1982); Baca y Stein (1983); Shils (1984); Passmore (1984); Robinson y Moulton (1985); Cahn (1990); May (1990); Counelis (1993). 
tan antes que nada un buen indicador de la atracción que suscitan las cuestiones éticas para una ciudadanía cada vez más escéptica ante la integridad pública (Natale, 1990 , p. 16). No es por ello raro que, paralelamente a lo que ha sucedido en el campo de la administración pública en general, los problemas relativos a la ética de la administración educativa y escolar, en sus diferentes aspectos, funciones y niveles (de la macro a la micropolítica) sean, a un lado y otro del Atlántico, objeto de una variada gama de estudios y análisis, muchos de las cuáles buscan trascender los planteamientos de regulación deontológica para situarse en la órbita de las nuevas discusiones en psicología y filosofía moral ${ }^{4}$. Tampoco aquí ha faltado la elaboración normativa. Numerosos distritos escolares y Juntas de Educación estadounidenses, al igual asociaciones profesionales como la National Association of Secondary School Principals o la Association of School Business Offcials, han establecido en las últimas décadas códigos o declaraciones de ética profesional. El precedente más significativo es el Código de Ética aprobado en 1966 por la American Association of School Administrators, un amplio texto dividido en dos grandes secciones: la primera formada por nueve principios generales con ejemplos de aplicación y un apartado final sobre supervisión, y la segunda dedicada a las medidas para la promoción de una conducta profesional ética.

La intervención, orientación y tratamiento psicopedagógico en sus distintas modalidades es otra de las áreas con una consolidada tradición ética y deontológica, en la que son recurrentes problemas tales como los que giran alrededor del principio de confidencialidad, situación de comunicación privilegiada (secreto profesional), deber de prevención y de protección a terceros, etc. ${ }^{5}$. En el aspecto normativo al margen del marco general de los Principios éticos de la American Psychological Association con sus diversas readaptaciones y de algunas elaboraciones de carácter más sectorial, hay que destacar textos como los de la American Scbool Counselor Association, de 1984, o la versión actualizada, de1988, del código de la American Association for Counseling and Development. Dentro del área general de la intervención psicopedagógica, se puede desgajar, por otro lado, un desarrollo específico en lo que se refiere a los problemas éticos de la educación especial $^{6}$. De hecho, tampoco aquí la pretensión normativa es un fenómeno nuevo. En 1983 el Council for Exceptional Children adoptaba un Código de ética y normas de práctica profesional, sucesor de otro menos difundido elaborado en 1964 siguiendo una intención cuyos primeros precedentes pueden situarse ya en los años veinte.

En 1975 un informe de Moeckli - entonces Secretario General de la Universidad Popular de Jura, en Suiza- publicado por el Consejo de Europa, apelaba a la necesidad de una deontología de los animadores socioculturales tanto vocacionales como profesionales (Moeckli, 1975). Pero, quizás, las mayores posibilidades

4. Puede verse: Strike, Haller y Soltis (1988); Beck (1992); Bottery (1992); Starrat (1991 y 1994).

5. Entre las aportaciones de los últimos años: Journal of Counseling and Development (1986); Jacob y Hartshorne (1991); Pope y Vázquez (1991); Schulte y Cocharne (1995).

6. Especialmente: Howe y Miramontes (1991 y 1992). 
en este área haya que buscarlas a través de la experiencia en los aspectos éticos y deontológicos de la profesión que viene acumulándose en diversos países en el campo vecino del trabajo social ${ }^{7}$. Con primeras tentativas que se remontan, como en otros casos, a los años veinte, asociaciones americanas de trabajadores sociales como la National Association of Black Social Workers, establecieron también a lo largo de la década de los ochenta códigos deontológicos. El texto más representativo es el código de la National Association of Social Workers, una asociación de ámbito federal formada en 1955 por fusión de organizaciones sectoriales que elaboró su primer código en 1960. Tras algunas modificaciones, una revisión en profundidad dio lugar al texto adoptado en 1979, el cual suscitó cierta polémica al verse en él una tendencia hacia lo individual frente a lo social, y ha sido objeto de nuevas revisiones en 1990 y $1993^{\circ}$.

La investigación pedagógica abre, por último, otra gran área que tanto en Estados Unidos como en Europa está suscitando atención ética y deontológica. No sólo confluyen en ella preocupaciones similares a las de otros sectores de investigación, sobre objetividad, comunicabilidad, fraude, etc. sino que su situación entre las ciencias humanas y sociales la dota de un especial significado ético, como bien refleja Helmut Danner:

"La idea de una teoría educativa en la que la responsabilidad existencial es un principio de conocimiento puede servir de paradigma para otras ciencias humanas, y en un sentido más amplio incluso para todas las ciencias en tanto que sus procedimientos afecten a los seres humanos y sus necesidades. El contenido de esta responsabilidad es hacer posible y servir de base a la mejor responsabilidad social y ética. Se trata de una responsabilidad social y ética para con el objeto de estudio y no sólo para con los procedimientos científicos. Su rango es el de la humanidad, el respeto y el cuidado por la personal..." (Danner, 1986, p. 37).

Dentro del marco tradicional de los aspectos éticos de la investigación en ciencias sociales, en los últimos años se ha desarrollado así, una literatura específica sobre la ética de la investigación pedagógica según los diferentes métodos y paradigmas de investigación?. Y, dentro del mismo marco, en el que las principa-

7. Véase: Dolgoff y Loewenberg (1985); Wells y Masch (1986); Lauffer (1987); Reamer (1990); Barker (1992); Reid y Popple (1992); Hugman y Smith (1995).

8. En el contexto internacional hay que hacer mención también la versión actual del Código de ética de la Federación Internacional de Trabajadores Sociales, mientras que en el europeo cabe señalar textos como las de la Britis Association of Social Workers (edición revisada de 1986), o la Association Nationale des Assitants de Service Social, recientemente en proceso de actualización. En lo que se refiere a nuestro país, el Colegio Profesional de Diplomados en Trabajo Social y Asistentes Sociales de Cataluña publicó en 1989 el primer código de ética profesional, instando a los demás colectivos profesionales a sumarse a esta iniciativa.

9. Una visión bastante comprensiva la proporciona la obra editada en el Reino Unido por Burguess (1989). Concretamente, sobre la ética en la investigación cualitativa y metodología etnográfica en educación son de gran interés varios de los trabajos contenidos en el libro editado por Eisner y Peshkin (1990); también en este sentido: Jover (1994b). 
les asociaciones profesionales de Estados Unidos en campos como la antropología, ciencia política, psicología o sociología han establecido prescripciones deontológicas referidas a la investigación, también la American Educational Research Association propuso en 1992 unas normas éticas que han difundido entre sus miembros con la intención de estimular el debate en este terreno.

En resumen, puede afirmarse que la deontología de las profesiones educativas cuenta hoy con grandes posibilidades de desarrollo tanto en un sentido extensivo (diversidad de áreas), como en un sentido intensivo (tradición dentro de cada área). Según Strike y Ternasky tres factores muy relacionados sirven para explicar el actual relanzamiento ético: a) la repercusión social que están adquiriendo los casos de conductas reprobables en la administración pública o en los negocios, y a la que no ha escapado la educación; b) la demanda de profesionalidad, que supone el reconocimiento de una mayor capacidad de autogobierno de los profesionales, y c) la caracterización de la actividad educativa en términos de "arte moral". Pero los mismos autores se manifiestan escépticos acerca de las posibilidades de las regulaciones deontológicas en educación, y destacan algunas de sus dificultades, como la escasa repercusión real que muchas veces tienen estos códigos en la práctica profesional, o la ausencia de una vinculación automática entre conocimiento y acción ética (Strike y Ternasky, 1993, pp. 2-5).

Tales dificultades revelan la necesidad de un mayor trabajo de fundamentación de la deontología de las profesiones educativas, que suele sufrir en general de la misma carencia que Reamer y Abramson denunciaban hace unos años en el campo del trabajo social:

"Hay literalmente sólo algunos estudios que aborden el tema de la ética del trabajo social de una forma disciplinada, sistemática, que tenga de alguna forma en cuenta la relación entre la tradición de pensamiento e ideas de la filosofía moral y los problemas éticos contemporáneos de la profesión. Lo que hay disponible en la literatura sobre trabajo social, tiende prácticamente a discusiones superficiales de valores tradicionales (si bien nadie duda de su importancia) tales como el respeto a la dignidad del cliente, la promoción de la autodeterminación y equidad, mantenimiento de la confidenciliadad, y otros similares. El número de artículos que discuten cuestiones acerca de la justificación de los valores del trabajo social, y los problemas que encuentran quienes realizan esta actividad al tratar de resolver conflictos entre ellos, es relativamente pequeño" (Reamer y Abramson, 1982, pp. 5-6).

La consecuencia más clara de esta ausencia de fundamentación, probablemente haya sido la de un excesivo afán reglamentista en medio de una gran ambigüedad sobre el significado y posibilidades reales de la deontología profesional. A mi juicio, en lo que se refiere a nuestro ámbito, tal pretensión de fundamentación exige considerar al menos tres tipos de argumentos, o aunar tres diferentes perspectivas de análisis: sociológica, ético-jurídica y pedagógica. 


\section{MARCO DE FUNDAMENTACIÓN DE LA DEONTOLOGÍA PROFESIONAL:}

Sociológicamente, el desarrollo de la deontología profesional va unido a las aspiraciones de profesionalización de las ocupaciones. De este modo, el interés por la deontología profesional en el terreno educativo se va a producir bajo el lema de la profesionalización. Ahora bien, como señala Abbott, al igual que existen distintas tendencias sociológicas para explicar el fenómeno y significado de la profesionalización, así hay también diferentes maneras de enfocar sociológicamente el sentido y objetivos de la deontología profesional y de los códigos deontológicos. Desde un enfoque funcionalista, las profesiones se justifican por su contribución a la satisfacción de necesidades sociales sobre la base de unos conocimientos y destrezas específicas, que dotan de autonomía al profesional, tanto en el ejercicio individual de su actividad como colectivamente a través de organizaciones profesionales. Los códigos deontológicos se entienden como instrumentos que velan por el correcto funcionamiento de la profesión mediante la autorregulación y autocontrol del cuerpo profesional. Frente a éste, desde un enfoque cooperativista o monopolista, la deontología profesional no se vincula tanto al control de la profesión como a su engrandecimiento, unido a motivaciones económicas, tensiones de poder, pugnas con otros colectivos profesionales, etc. El código deontológico cumple básicamente un cometido de conglomeración y demarcación profesional hacia el interior y hacia el exterior de la profesión. La apelación en los códigos a la relación con los colegas o a los requisitos para el ejercicio profesional servirán fundamentalmente a este propósito.

Abbott considera que es posible distinguir un tercer enfoque, con entidad propia suficiente, centrado en el status profesional que confiere la deontología a través de la apelación a la idea de servicio, desinterés, etc. El papel de los códigos deontológicos sería aquí el de dotar de prestigio e imagen social a la profesión. Según este autor, estos tres enfoques no son teorías alternativas entre las que haya que elegir, sino que en un fenómeno complejo como la deontología profesional están envueltos varios efectos al mismo tiempo. La historia de la deontología profesional puede entenderse desde aquí como la historia de las tensiones entre esos tres efectos. Cada uno de ellos tendrá más fuerza en aquellas situaciones en las que las posibilidades de estrategias alternativas para alcanzarlos decrezcan. Así, en aquellos momentos en los que las profesiones experimentan un déficit de status, es lógico esperar que se produzca una expansión de la deontología profesional enfocada hacia este efecto, tal como sucedió en Estados Unidos en los años 20 (Abbot, 1983).

Pues bien, en esta línea, la situación en la que parece producirse la nueva apelación a la deontología profesional y proliferación de instrumentos normativos, sobre todo a partir de la década de los ochenta, creo que puede caracterizarse, según sugerí al principio, como la de una pérdida de confianza. Como notaba House al comienzo de la década:

"Desde hace algún tiempo, se observa en el público en general la tendencia a negar a los profesionales el derecho a controlar sus propios asuntos. Los usuarios demandan un puesto en los consejos directivos de los 
centros de salud, en los consejos asesores y en los órganos de dirección de las escuelas. Existe la creencia de que los profesionales no vigilan sus propias operaciones con suficiente atención (...).

La desconfianza pública ante las evaluaciones controladas por los propios profesionales se refleja en la disminución de la credibilidad de los organismos encargados de la acreditación profesional (...) El control político de las actividades de reconocimiento y acreditación va pasando a manos de los gobiernos estatales" (House, 1994, p. 221) ${ }^{10}$.

En tal contexto, los códigos deontológicos vienen a cumplir sobre todo una función de legitimación y garantía, de intento, especialmente en las nuevas profesiones, de abrirse un espacio social y fomentar la imagen y confianza pública en la profesión. No es difícil hacer también esta interpretación del desarrollo en regulaciones deontológicas que han emprendido asimismö a lo largo de estos años algunas nuevas profesiones en nuestro país. Los códigos se presentan como signos de madurez profesional que colaboran a la implantación de la profesión, mostrando públicamente la disposición de los profesionales a regular su conducta según principios éticos, como se decía con ocasión de la aprobación de uno de estos códigos (Colegio Oficial de Psicólogos, 1987, p. 1); instrumentos que contribuyen a mantener un nivel profesional y un status social digno de la profesión, según se dice en otro de ellos (Organización Colegial de la Enfermería, 1989, p. 7).

En lo que se refiere más específicamente a la profesión docente, es sobradamente conocida la sensación que tienen los profesores de no ser suficientemente valorados socialmente, tal como ilustran diversas investigaciones e informes nacionales e internacionales ${ }^{11}$. Y también aquí se ha utilizado ya el recurso de la deontología. Cuando en 1966 la U.N.E.S.C.O. recomendaba a las organizaciones profesionales de profesores elaborar normas de conducta ética, lo hacía porque "dichas normas contribuyen en gran parte a asegurar el prestigio de la profesión y el cumplimiento de los deberes profesionales según principios aceptados" (U.N.E.S.C.O., 1966, art. 73) ${ }^{12}$. Lógicamente, la condición para esa recuperación de la imagen y confianza pública, es que el propio código cuente con suficiente credibilidad social, lo que explica que las organizaciones profesionales hayan optado por abrirse a la participación del público en el tratamiento de las cuestiones deontológicas, pues, como escribe Rich, ula consecuencia para la profesión que niega tal participación puede ser el rechazo del público a reconocer su status como profesión; negar la participación, puede, a la larga, llevar a demandas de mayor regulación externa a la profesión" (Rich, 1984, p. 53). Se considera, así, ahora que la discusión sobre problemas deontológicos no siempre implica la posesión de un conocimiento profesional experto, sino que a menudo se refiere a problemas y

10. Versión original de 1980.

11. Véase, por ejemplo: OCDE (1990, pp. 43-68); Nave (1992, pp. 114-135); González Blasco y González-Anleo (1993, pp. 126-128); Guerrero (1993, pp. 95-96).

12. La cursiva es mía. 
decisiones que caen fuera o están más allá de este conocimiento, exigiendo, en consecuencia, la apertura al público (Byles, 1981, pp. 138-139).

Normativamente, los códigos deontológicos tienen un estatuto peculiar, siendo habitual situarlos en el espacio intermedio entre lo jurídico y lo ético. La positivación en una norma, procesos formales de adopción, carácter vinculante para los miembros del colectivo profesional, etc. los dota de cierta naturaleza jurídica. Su operatividad como tal suele ser puesta, sin embargo, en entredicho, al no poder garantizarse sistemas adecuados de seguimiento y control, ni en última instancia la preeminencia del interés de los destinatarios de la actividad. Y es probable que la razón sea, justamente, ese aludido carácter intermedio, que deja a los códigos en una zona de gran ambigüedad, ni estrictamente lo uno ni totalmente lo otro. Ante tal ambigüedad, la primera alternativa es acentuar su función de control externo, de ley, y de ello hay ya experiencias en el caso específico de la actividad docente, como la desarrollada en los años ochenta en el Estado norteamericano de Florida, en el que se dotó de rango legal al código deontológico, estableciéndose un mecanismo "cuasi-judicial" para su control, a través de un comité formado por cinco profesores, cinco miembros de la administración y tres particulares. No obstante, MacMillan se pregunta, hasta qué punto este sistema no refuerza aún más el mensaje de falta de confianza (MacMillan, 1993, pp. 197-200).

Frente a ésta, la segunda alternativa es otorgar la primacía al carácter ético del código, a su sentido de obligación moral autoasumida. En este segundo caso el problema de la operatividad reconduce al de la fundamentación ética de los códigos: ¿qué es lo que hace de los códigos y sus enunciados fácticos un conjunto de prescripciones morales? ¿basta con acudir a criterios procedimentales? ¿en qué se basa finalmente su obligatoriedad moral, es decir, con independencia de cualquier consecuencia legal?... Lo que anda en juego es, ni más ni menos, que el problema de la fundamentación de los criterios éticos.

No es casualidad que la proliferación de regulaciones deontológicas se produzca, precisamente, en un momento de gran incertidumbre, en el que la ética se encuentra ante la disyuntiva que Bernstein plantea al comienzo de la obra Habermas y la modernidad: «Podemos conseguir todavía, en nuestra época, una justificación racional de los estándares normativos universales? ¿O nos enfrentamos con el relativismo, decisionismo, o el emotivismo que defienden que las normas son en último término arbitrarias y trascienden una justificación racional? (Bernstein, 1991, p. 18). Como trata de mostrar MacIntyre en un trabajo sobre deontología empresarial, los dilemas morales en el terreno de la ética profesional, provienen de esa misma inseguridad, cuya raíz hay que situar en la pervivencia y enfrentamiento de ideales y concepciones éticas diferentes. Nuestros argumentos morales son retazos que tienen su origen en distintas tradiciones, lo que los hace difícilmente confrontables. Ello, unido a la necesidad de adoptar elecciones y decisiones morales, hace que inevitablemente nos tengamos que enfrentar al conflicto, el desacuerdo y los problemas irresolubles. Carecemos hoy de un criterio claro para la resolución de tales dilemas éticos, y debemos acostumbrarnos a vivir y trabajar en la incertidumbre moral (MacIntyre, 1983) ${ }^{33}$.

13. Para un desarrollo de estos argumentos, véase también: Maclntyre (1994). 
Para quienes, parafraseando a Heller y Fehér, preferimos pensar que cuestiones como el genocidio no son simplemente un asunto de gusto (Heller y Fehér, 1989, p. 159), una posible salida la ofrece la idea de los derechos humanos. El propio Macintyre parece cerrar esa opción cuando señala en que la mejor prueba en contra de la misma es que nadie ha probado hasta ahora la existencia de tales derechos, y creer en ellos es como creer en brujas y unicornios (MacIntyre, 1987, p. 95). Pero la crítica de MacIntyre no se refiere tanto a los derechos en sí como a una concepción apriorística de los mismos. Como hemos propuesto en otro lugar, cabe todavía pensar en ellos como aspiraciones y expectativas sociomorales que se fraguan al hilo de la historia, en una acepción que si rechaza un apriorismo ético, tampoco los reduce sólo a su acepción de categoría jurídica (Jover, 1994a, pp. 45-48; Bárcena, Gil y Jover, en prensa). Los mismos podrían proporcionar entonces una base ética a la deontología profesional, y a la deontología en el campo de las profesiones educativas.

Sin embargo, no es sólo ilusorio, sino también contraproducente, aspirar a la posibilidad de un criterio moral de aplicación inmediata a la resolución de cualquier problema ético. Y sorprende que ese afán reglamentista en ética profesional se produzca también en un momento en el que, ante el desencanto de las grandes construcciones normativas de validez incondicional, la ética parece volver a aferrarse otra vez más a la noción de un juicio práctico que se ejerce en situación. Es posible, por ejemplo, hacer esta lectura de la reconstrucción falibilista de la ética habermasiana que realiza Wellmer desde lo que él mismo denomina una óptica cuasikantiana, tan a partir de Kant como frente a Kant, en la que el progreso moral no se sitúa ya en el logro del sentido o de aproximación al ideal, sino en la negación del sinsentido, y el juicio moral se entiende como un juicio sobre la generalidad o no generalidad de maneras-de-actuar-en-situaciones, que sólo puede fundamentarse recurriendo al análisis de las situaciones específicas y convierte la pregunta acerca de la acción moral en ula pregunta acerca de una comprensión adecuada de las situaciones accionales concretas" (Wellmer 1994, p. 152).

Pues sucede que también en el terreno de la deontología profesional los verdaderos problemas "comienzan con el de la mediación entre lo particular y lo general" (Wellmer 1994, p. 144), por lo que, si bien los códigos deontológicos pueden servir de guías y marcos de expectativas, representan sólo una ayuda limitada para la resolución de dilemas morales en situaciones profesionales, al tener que moverse necesariamente en un ámbito de elevada generalidad, so pena de caer en la trivialidad. A lo que habría que añadir la dificultad que supone el salto entre conocimiento y acción, razón de que, en el plano ético más aún que en el jurídico, la simple existencia y conocimiento del código no garantice la conducta acorde. La apelación al juicio del profesional, a su decisión siempre arriesgada, más allá de la aspiración a certezas últimas, parece, en consecuencia, ineludible. Ya lo decía Aristóteles, pretender en estos casos la seguridad del matemático no es propio de un hombre instruido (Et. Nic., 1,3, 1094b).

El propio énfasis en las regulaciones deontológicas puede considerarse desde este punto de vista como un aspecto de lo que Soder denomina la retórica de la profesionalización (Soder, 1990 y 1991). Una retórica que choca a menudo con las 
condiciones reales de ejercicio (Apple y Jungck, 1992), y de cuyas contradicciones puede ser un signo más ese mismo afán reglamentista. Lo sugiere la crítica de Marañón cuando, refiriéndose a la ética médica, decía que el buen profesional no necesita de reglamentos para su rectitud, y a quien no lo es las reglas y consejos morales le resultan perfectamente inútiles; "Sobran aquí, como en todos los problemas de conducta moral, las leyes" (Marañón, 1981, p. 62). La duda, por tanto, es: ¿ese afán reglamentista, no puede entrar en contradicción con la misma idea de profesionalización a la que dice servir? La pregunta involucra múltiples problemas, relativos, por un lado, a los diferentes efectos que pueden pretenderse con el recurso a la deontología profesional y la posible colisión entre las condiciones que cada uno de ellos exige; pero también, por otro, en lo que se refiere más directamente a nuestro caso, a la propia naturaleza del conocimiento pedagógico y de la actividad educativa.

La fundamentación pedagógica de la deontología profesional exige, en efecto, tener en cuenta la peculiar dimensión o significado ético implícito en el concepto de actividad educativa. En nuestra aportación al debate que bajo el título de "Moral profesional: dimensiones éticas de la enseñanza" dedicó al tema la revista The Journal of Moral Education, nos propusimos así revełar las inconsistencias de los argumentos que más frecuentemente se proponen para justificar tal dimensión, tratando de poner de manifiesto como el planteamiento de regulación deontológica es insuficiente para una comprensión en profundidad de la misma. A nuestro juicio, desde un punto de vista formal, se atiende a dicha dimensión cuando, a lo largo del proceso educativo, se planifica la ayuda pedagógica de forma que se incida en la estructura moral del educando en orden a suscitarle efectos de orientación social y personal, mientras que como contenido de dicha dimensión se sugiere la idea de una socialización basada en los derechos humanos, en el sentido mencionado de aspiraciones morales históricas (Bárcena, Gil y Jove, 1993).

Bajo esta propuesta subyace la pretensión de una reelaboración del concepto de eficacia pedagógica, que se alarga para incluir las aspiraciones sociomorales, $y$ en el que existe una separación tajante entre medios y fines, entre forma y contenido, por lo que la propia situación en la que se desarrolla la actividad se convierte en un escenario pedagógico. Y es desde aquí desde donde cabe entender mejor el significado pedagógico que puede adquirir el código deontológico: su contribución a la generación de un cierto clima educativo. Se trata, por tanto, de entender el código como recurso pedagógico, en un sentido más radical del de ser simplemente un instrumento para el aprendizaje de deberes profesionales.

Es muy sugerente a este respecto la propuesta que, con distintas formulaciones, ha venido realizando Sockett en el terreno de la profesión docente (Sockett, 1985, 1990 y 1993). Según este autor, los códigos deontológicos tradicionales instituidos por asociaciones profesionales como marcos regulativos generales, adolecen en esta profesión de posibilidades y operatividad. Por ello sugiere acercarlos más a la práctica y a los problemas y preocupaciones reales mediante su formulación a nivel local, o incluso escolar, de modo que representen un compromiso más auténtico de los implicados. Un código así vendría a servir de documento de consulta y guía, acorde con un sentido de la profesionalidad en la que se rinde 
cuenta y se es responsable fundamentalmente ante la propia comunidad educativa en la que se ejerce. Pero su principal valor pedagógico, como señala el propio Sockett, radica en su virtualidad de configuración de un determinado ethos escolar, al fomentar un estilo de relaciones interpersonales basadas en la confianza. Para ello, el código debe cumplir algunos requisitos. Debe ser público y plasmar los distintos intereses. Debe ser manejable, esto es, convertirse en lugar de referencia en la resolución de problemas, sin por ello pretender anular la necesidad de interpretación y decisión... En definitiva, tiene que responder a situaciones reales, por lo que su fuente principal debe ser la propia experiencia de quienes participan en su formulación (Sockett, 1993, pp. 108-127).

Creo, para finalizar, que esta propuesta de Sockett es bastante digna de consideración en nuestro caso, sobre todo a la vista de la autonomía como factor de calidad que se desea para las instituciones escolares, y de la pretensión de que "Cada centro tenga su propia personalidad definida en el proyecto educativo" y un esquema claro "de las relaciones entre todos los miembros de la comunidad educativa ${ }^{14}$. Nada impediría, por otra parte, que un código de este tipo representase la especificación de un marco regulativo más amplio. Pero sobre todo en un ambiente de escepticismo y "crisis de representación", es importante que el código se base en la propia experiencia de quienes participan en una misma situación educativa. El código como expresión de ideales y objetivos compartidos; elemento de un proyecto educativo.

14. Real Decreto 819/1993, de 28 de mayo, por el que se aprueba el Reglamento Orgánico de las Escuelas de Educación Infantil y de los Colegios de Educación Primaria, préambulo (B.O.E., 19-6-1993). 


\section{BIBLIOGRAFÍA}

Aввот, A. (1993): Professional Ethics, American Journal of Sociology, vol. 88, $\mathrm{n}^{\circ}$ 5, pp. $855-885$.

APple, M.W., y JungCK, S. (1992): You don't have to be a Teahcer to Teach this Unit: Teaching, Technology and Control in the Classrrom. En Hargreaves, A. y Fullan, G. (Eds.) Uniderstanding Teacher Development. New York, London, Teachers College Press, pp. 20-42.

BACA, M.C. y STEIN, R.H. (Eds.) (1983): Etbical Principles. Practices and Problems in Higher Education. Springfield, Charles C. Thomas.

Barcena, F.; Gil F. y Jover, G. (1993): The Ethical Dimension of Teaching: a review and a proposal, The Jorunal of Moral Education, vol 22, $\mathrm{n}^{\circ} 3$, pp. 241-252.

BARCENA, F.; GIL F. y Jover, G. (en prensa): La socialización como forma de educación moral: Una propuesta en el contexto de la reforma educativa, Pedagogía Social. Revista Interuniversitaria.

BARKER, R.L. (1992): Social Work in Private Practice. Washington, National Association of Social Workers Press.

BAYLERS, M.D. (1981): Professional Ethics. Belmont. Wadsworth.

BeCK, L.G. (1992): Meeting the Challenge of the Future: The Place of a Caring Ethic in Educational Administration, American Journal of Educatin, vol. 100, no 4, pp. 454-496.

Bernstein, R.J. (1991): Introducción. En: Guiddens, A. et al. Habermas y la modernidad, Madrid, Cátedra, pp. 13-61.

BOTTERY, M. (1992): The Ethics of Educational Management. London, Cassell.

Burgess, R.G. (Ed.) (1989): The Ethics of Educational Research. New York, Philadelphia, London, The Falmer Press.

CAHn, S.M. (Ed.) (1990): Morality. Responsability and the University. Philadelphia, Temple University Press.

Camps, V. (1990): Virtudes públicas. Madrid, Espasa-Calpe.

Colegro Oficial de Psicólogos (1987): Código deontológico: autorregulación de la profesión: defensa del usuario, Papeles del Colegio de Psicólogos, vol. 6, no 32, p. 1.

COMISIÓN DE LAS COMUNIDADES EUROPEAS (1993): Orientaciones para la acción comunitaria en el ámbito de la educación y la formación. Luxemburgo, Oficina de Publicaciones Oficiales de las Comunidades Europeas.

Consejo Escolar Catalán (1993): Criterios para una deontología del docente, boletín del Colegio de Doctores y Licenciados, abril, pp. 5-18.

Consejo General de Colegios Oficiales de Doctores y LiCEnciados en Filosofía y letras y en Ciencias (1994): Borrador de Código Deontológico, Boletín del Colegio de Doctores y Licenciados, febrero, pp. 19-20.

CounELIS, J.S. (1993): Toward Empirical Studies on University Ethics, The Journal of Higher Education, vol. 24, no 1 , pp. 74-92.

Danner, H. (1986): Human Science is Responsable, Phenomenology + Pedagogy, vol. 4, $\mathrm{n}^{\circ}$ 1 , pp. $32-40$.

Dolgoff, R. y Loewenberg, F. (1985): Ethical Decisions for Social Work Practice. Itasca, F. E. Peacock.

EISNER, W.W. y PeshKin, A. (Eds.) (1990): Qualitative Inquiry in Education. New York, London, Teachers College Press.

González Blasco, P y González-ANleo, J. (1993): El profesorado en la España actual. Madrid, S.M.

GOODlad, J.I.; SODER, R. y SirotniK, K.A. (Eds.) (1990): The Moral Dimensions of Teaching. San Francisco, Oxford, Jossey-Bass. 
Guerrero, A. (1993): El magisterio en la Comunidad de Madrid. Madrid, Consejería de Educación y Cultura de la Comunidad de Madrid.

Heller, A. y Feher, F. (1989): Politicas de la postmodernidad, Barcelona, Península.

HERRING, M.Y. (1988): Ethics and the Professor. An Annotated Bibliography. 1970-1985. New York, London, Garland.

Hortal, A. (1994): Planteamiento de una ética profesional. En: Fernández Fernández, J.L. y Hortal Alonso, A. (Eds.). Etica de las profesiones. Madrid, UPCO, pp. 55-73.

House, E.R. (1994): Evaluación, ética y poder. Madrid, Morata.

Howe, K.R. y Miramontes, O.B. (1991): A Framework for Ethical Deliberation in Special Education, Journal of Special Education, vol. 25, no 1, pp. 7-25.

Howe, K.R. y Miramontes, O.B. (1991): The Ethics of Special Education. New York, London, Teachers College Press.

Hugman, R. y SMTth, D. (1995): Ethical Issues in Social Work. Londond, Routledge.

JACOB, S. y HARTSHORne, T. (1991): Ethics and Law for School Psychologists. Brandon, Clinical Psychology Pub.

Journal of Counseling and Development, (1986): vol. 64, $\mathrm{n}^{\circ} 5$ (Número monográfico sobre ética profesional).

The Journal of Higher Education (1982): vol. 53, $\mathrm{n}^{\circ} 3$, Ethics and the Academic Profession.

The Jornal of Moral Education (1993): vol. 22, $\mathrm{n}^{\circ} 3$, Special Issue: Professional Morality. Ethical Dimensions of Teaching.

Journal of Teacher Education (1986): vol. 37, no 3, Profesional Ethics in Teacher Education.

Journal of Teacher Education (1991): vol. 42, $\mathrm{n}^{\circ} 3$, The Ethical Responsabilities of Teaching.

Journal of Thought, (1987): vol. 2, $\mathrm{n}^{\circ} 3$, Ethics and Education.

Jover, G. (1991): Ámbitos de la deontología profesional docente, Teoría de la Educación. Revista Interuniversitaria, vol. 3, pp. 75-92.

Jover, G. (1994a): Ámbito de la Política de la Educación. En: Colom, A. (Ed.), Política y planificación educativa, Sevilla, Preu Spinola, pp. 41-50.

Jover, G. (1994b): La cuestión ética en la investigación etnográfica. XIII Seminario Interuniversitario de Teoría de la Educación "Antropología de la Educación", comunicaciones, vol. II.

Kultgen, J. (1988): Ethics and Professionalism. Philadelphia, University of Pennsylvania Press.

Lauffer, A. (1987): Working in Social Work. Newbury Park, Sage Publications.

LIPOVETSKY, G. (1994): El crepúsculo del deber, Barcelona, Anagrama.

MacIntyre, A. (1983): Why are the Problems of Business Ethics Insoluble? En: Baumrin, B. y FreEdman, B. (Eds.) Moral Responsibility and the Professions. New York, Haven Publications, pp. 350-359.

MaCINTYRe, A. (1987): Tras la virtud. Barcelona, Crítica.

MACINTYRE, A. (1994): Justicia y racionalidad. Barcelona, Ediciones Internacionales Universitarias.

MacMillan, C.J.B. (1993): Ethics and Teacher Professionalization. En: STRIKE, K.A. y Ternasky, P.L. (Eds.) Ethics for Professionals in Education. New York, London, Teachers College Press.

MARAÑón, G. (1981): Vocación y ética y otros ensayos. Madrid, Espasa-Calpe.

MAY, W.W. (Ed.) (1990): Ethcis and Higher Education. New York, American Council of Education, MacMillan.

MoECKLI, J.M. (1975): La déontologie des animaterus,Bulletin d'Information (Conseil de l'Europe), $\mathrm{n}^{\circ} 4$, pp. 73-81.

NATALE, J.A. (1990): School Board Ethics: On Thin Ice? The American School Board Journal, vol. $117, \mathrm{n}^{\circ} 10$, pp. $16-19$. 
Nave, G. (1992): The Teaching Nation, Propects for Teachers in the European Community. Oxford, New York, etc., Pergamon Press.

O.C.D.E. (1990): The Teacher Today. Paris, OCDE.

Organización Colegial de la EnFermería (1989): Código deontológico de la enfermería española. Madrid, Rivadeneyra.

PASSMORE, J. (1984): Academic Ethics?, Journal of Applied Philosophy, vol. 1, no 1, pp. 63-77.

PoPe, K.s, y VASQuez, M.J.T. (1991): Ethics in Psychoterapy and Counseling. A Practical Guide for Psychologists. San Francisco, Oxford, Jossey-Bass.

Reamer, F.G. (1990): Ethical Dilemmas in Social Service. New York, Columbia University Press.

Reamer, F.G. y Abramson, M. (1982): The Teaching of Social Work Ethics. New York, The Hastings Center.

REID, P.N. y POPPLE, P.R. (1992): The moral Purposes of Social Work: The Character and Intentions of a Profession. Chicago, Nelson-Hall.

Rich, J.M. (1984): Professional Ethics in Education, Springfield, Charles C. Thomas.

Robinson, G.M. y Moulton, J. (1985): Ethical Problems in Higher Education. Englewood Cliffs, Preintice-Hall.

SCHulte, J.M. y Cocharne, D.B. (1995): Ethics in School Counseling, New York, London, Teachers College Press.

ShiLs, E. (1984): The Academica Ethics. Chicago University Press.

SOCKETT, H. (1985): Toward a Professional Code in Teaching. En: Gordon, P. (Ed.) Is Teaching a Profession. University of London, Instituye of Education, Heinemann, pp. 26-43.

SOcketT, H. (1990): Accountability, Trust and Ethical Codes. En: Goodlad, J.I.; SODER, R. y Sirotnik, K.A. (Eds.): The Moral Dimensions of Teaching. San Francisco, Oxford, Jossey-Bass, pp. 224-250.

SOCKeтt, H. (1993): The Moral Base For Teacher Porfessionalism. New York, London, Teacher College Press.

SODER, R. (1990): The Rhetoric of Teacher Professionalization. En: Goodlad, J.I.; SODER, R. y SiRotniK, K.A. (eds.): The Moral Dimensions of Teaching. San Francisco, Oxford, Jossey-Bass, pp. 35-86.

SODER, R. (1991): The Ethics of the Rhetoric of Teacher Professionalization, Teaching and Teacher Education, vol. 7, n ${ }^{\circ}$ 3, pp. 295-302.

StARRATT, R. J. (1991): Building an Ethical School: A Theory for Practice in Educational Leadership, Educational Administration Quarterly, vol. 27, n 2, pp. 185-202.

StarRatT, R. J. (1994): Building an Ethical Scbool. London, New York, Philadelphia, The Falmer Press.

StRIKe, K.A.; Haller, E.J. y Soltis, J.F. (1988): The Etichs of School Administration. New York, London, Teachers College Press.

StriKe, K.A. y Ternasky, P.L. (1993): Etbics in Educational Settings. En: STRIKe, K.A. y TerNASKY, P.L. (Eds.) Ethics for Professionals in Education. New York, London, Teachers College Press.

StRIKE, K.A. y TeRnasky, P.L. (Eds.) (1993): Ethics for Professionals in Education. New York, London, Teachers College Press.

U.N.E.S.C.O. (1996): Recomendación relativa a la situación del personal docente. En ComISIÓN NACIONAL ESPAÑOLA DE COOPERACIÓN CON LA UNESCO (1981): Convenciones, Recomendaciones y Declaraciones de la UNESCO. Madrid, M.E.C.

Wellmer, A. (1994): Etica y diálogo, Barcelona, Anthropos.

Wells, C.C. y Masch, M.K. (1986): Social Work Ethics Day to Day. New York, London, Longman. 